\title{
Molecular Dynamics and Density Functional Studies of Substrate Binding and Catalysis of Arginine Deiminase
}

Canhui Wang, ${ }^{1}$ Dingguo $\mathrm{Xu},{ }^{1}$ Lidong Zhang, ${ }^{2}$ Debra Daiqian Xie, ${ }^{2, *}$ and Hua Guo ${ }^{1, *}$

${ }^{1}$ Department of Chemistry, University of New Mexico, Albuquerque, NM 87131, USA

${ }^{2}$ Institute of Theoretical and Computational Chemistry, Key Laboratory of

Mesoscopic Chemistry, School of Chemistry and Chemical Engineering, Nanjing

University, Nanjing 210093, China

\section{Supplement Information}

Coordinates of stationary points in three DFT models.

$\begin{array}{lrrr}\text { Model A_RC } & & & \\ \mathrm{C} & 0.00000000 & 0.00000000 & 0.00000000 \\ \mathrm{H} & 1.09337231 & 0.00000000 & 0.00000000 \\ \mathrm{H} & -0.35557897 & 1.03359705 & 0.00000000 \\ \mathrm{~N} & -0.52398617 & -0.74787955 & -1.14232761 \\ \mathrm{H} & -1.14033502 & -1.53489145 & -0.95638436 \\ \mathrm{C} & -0.22433384 & -0.49249644 & -2.41998332 \\ \mathrm{~N} & 0.51884325 & 0.56022547 & -2.76112731 \\ \mathrm{H} & 0.79269835 & 0.66530031 & -3.72751005 \\ \mathrm{H} & 0.85261011 & 1.31660349 & -2.11529990 \\ \mathrm{~N} & -0.67310405 & -1.33677748 & -3.37544474 \\ \mathrm{H} & -0.61866388 & -1.08483179 & -4.34998308 \\ \mathrm{H} & -1.23126219 & -2.14630846 & -3.13499942 \\ \mathrm{H} & -0.35052123 & -0.49232301 & 0.90684072 \\ \mathrm{C} & -2.34963797 & -5.21950677 & -0.60187310 \\ \mathrm{H} & -2.96332441 & -5.96182509 & -1.11229247 \\ \mathrm{H} & -1.29683411 & -5.47920080 & -0.72176806 \\ \mathrm{~S} & -2.56889904 & -3.57230881 & -1.38522285 \\ \mathrm{H} & -3.88450420 & -3.39835615 & -1.15987478 \\ \mathrm{H} & -2.59471739 & -5.17534380 & 0.45935587 \\ \mathrm{H} & 3.54728169 & 1.95071480 & -0.87726542 \\ \mathrm{~N} & 1.60117403 & 2.75245478 & -1.35311624 \\ \mathrm{C} & 2.90497953 & 2.81881506 & -0.89642069 \\ \mathrm{C} & 1.11667095 & 3.98014768 & -1.24686140 \\ \mathrm{H} & 0.12079044 & 4.29805305 & -1.51983355 \\ \mathrm{H} & 2.05085181 & 4.82582983 & -0.74015778 \\ \mathrm{H} & 1.92538801 & 5.81351004 & -0.56710335 \\ & 3.20127990 & 4.10065521 & -0.51036031 \\ \mathrm{H} & -09673895 & 4.54786268 & -0.10847217\end{array}$




$\begin{array}{crrc}\text { ModelA_TS1 } & & & \\ \mathrm{C} & 0.00000000 & 0.00000000 & 0.00000000 \\ \mathrm{H} & 1.08974810 & 0.00000000 & 0.00000000 \\ \mathrm{H} & -0.37094802 & 1.03202789 & 0.00000000 \\ \mathrm{~N} & -0.51256150 & -0.71547338 & -1.17497577 \\ \mathrm{H} & -1.47653836 & -1.01583712 & -1.07862012 \\ \mathrm{C} & -0.16193268 & -0.41362142 & -2.43966580 \\ \mathrm{~N} & 1.23781036 & -0.20539716 & -2.77288259 \\ \mathrm{H} & 1.52349332 & -0.81033086 & -3.54813752 \\ \mathrm{H} & 1.98598133 & -0.32990142 & -1.98060210 \\ \mathrm{~N} & -0.91155147 & -0.89123430 & -3.46760260 \\ \mathrm{H} & -0.64389328 & -0.62837593 & -4.40726145 \\ \mathrm{H} & -1.91512722 & -0.93953390 & -3.34130319 \\ \mathrm{H} & -0.34542472 & -0.52783673 & 0.88916372 \\ \mathrm{C} & -1.12770819 & 2.49908255 & -4.14817209 \\ \mathrm{H} & -1.60658286 & 1.58087923 & -4.51119549 \\ \mathrm{H} & -1.90971501 & 3.22258827 & -3.91196098 \\ \mathrm{~S} & -0.12376043 & 2.19011820 & -2.64521994 \\ \mathrm{H} & 1.11151766 & 0.93948628 & -3.03407970 \\ \mathrm{H} & -0.50088898 & 2.90501022 & -4.94515762 \\ \mathrm{H} & 3.34245327 & -2.57818830 & -0.57576835 \\ \mathrm{~N} & 3.32054750 & -0.47018951 & -1.04597799 \\ \mathrm{C} & 3.85064010 & -1.62813491 & -0.50373105 \\ \mathrm{C} & 4.19126913 & 0.49596313 & -0.78220228 \\ \mathrm{H} & 4.09475957 & 1.53500108 & -1.06259728 \\ \mathrm{~N} & 5.24901047 & 0.00405989 & -0.09415998 \\ \mathrm{H} & 6.04576654 & 0.53863369 & 0.22496875 \\ \mathrm{C} & 5.05131592 & -1.34833598 & 0.09343969 \\ \mathrm{H} & 5.76036479 & -1.96982577 & 0.61713110\end{array}$




$\begin{array}{lrrr}\text { ModelA_INT } & & & \\ \mathrm{C} & 0.00000000 & 0.00000000 & 0.00000000 \\ \mathrm{H} & 1.09778804 & 0.00000000 & 0.00000000 \\ \mathrm{H} & -0.34533794 & 1.03552500 & 0.00000000 \\ \mathrm{~N} & -0.59207394 & -0.72162439 & -1.12715316 \\ \mathrm{H} & -0.57521098 & -1.73176453 & -1.02834841 \\ \mathrm{C} & -0.42912715 & -0.32032792 & -2.46663981 \\ \mathrm{~N} & 1.06805143 & -0.36984333 & -2.94165352 \\ \mathrm{H} & 1.38019668 & -1.33979915 & -2.84063288 \\ \mathrm{H} & 1.76613781 & 0.31021894 & -2.41439266 \\ \mathrm{~N} & -1.09882865 & -1.27670462 & -3.30062169 \\ \mathrm{H} & -1.21206373 & -0.96006499 & -4.25947072 \\ \mathrm{H} & -2.01110251 & -1.51381440 & -2.92239743 \\ \mathrm{H} & -0.34760233 & -0.47665409 & 0.91834112 \\ \mathrm{C} & -2.62074984 & 1.47552693 & -2.38112203 \\ \mathrm{H} & -2.79571589 & 1.12596420 & -1.36244738 \\ \mathrm{H} & -2.94683682 & 2.51380249 & -2.46973923 \\ \mathrm{~S} & -0.83501725 & 1.48438763 & -2.76346045 \\ \mathrm{H} & 1.12730901 & -0.13739417 & -3.93734588 \\ \mathrm{H} & -3.17672683 & 0.87042846 & -3.09964831 \\ \mathrm{H} & 4.60587572 & -0.01961518 & -1.68638671 \\ \mathrm{~N} & 2.89215753 & 1.29054270 & -1.81932381 \\ \mathrm{C} & 4.21558464 & 0.97927869 & -1.55985534 \\ \mathrm{C} & 2.75750624 & 2.58530589 & -1.56502381 \\ \mathrm{H} & 1.84715453 & 3.15814557 & -1.66378806 \\ \mathrm{H} & 3.93606552 & 3.11047788 & -1.15296891 \\ \mathrm{H} & 4.09583020 & 4.07622245 & -0.90010567 \\ \mathrm{H} & -87851468 & 2.10302063 & -1.14287448 \\ \mathrm{H} & 2.27086463 & -0.85016626\end{array}$




$\begin{array}{crrc}\text { ModelA_TS2 } & & & \\ \mathrm{C} & 0.00000000 & 0.00000000 & 0.00000000 \\ \mathrm{H} & 1.09511867 & 0.00000000 & 0.00000000 \\ \mathrm{H} & -0.37179332 & 1.02776257 & 0.00000000 \\ \mathrm{~N} & -0.53377830 & -0.73768486 & -1.14722910 \\ \mathrm{H} & -0.83578516 & -1.69097444 & -0.98580453 \\ \mathrm{C} & -0.33363613 & -0.42760089 & -2.44388308 \\ \mathrm{~N} & 1.40072949 & -1.25599795 & -2.96199269 \\ \mathrm{H} & 1.25079962 & -2.26456378 & -2.98027250 \\ \mathrm{H} & 2.12412564 & -1.02308680 & -2.24383328 \\ \mathrm{~N} & -1.14261199 & -1.18407474 & -3.30884784 \\ \mathrm{H} & -0.96363209 & -1.04868358 & -4.29603670 \\ \mathrm{H} & -2.13166208 & -1.07504914 & -3.10201532 \\ \mathrm{H} & -0.35754552 & -0.48977915 & 0.90608884 \\ \mathrm{C} & -0.17323922 & 1.48072828 & -4.52450017 \\ \mathrm{H} & -1.19096675 & 1.24894580 & -4.84359672 \\ \mathrm{H} & 0.02265126 & 2.53391497 & -4.73784135 \\ \mathrm{~S} & 0.01929606 & 1.33240897 & -2.71689256 \\ \mathrm{H} & 1.76421034 & -0.97852856 & -3.87309424 \\ \mathrm{H} & 0.55823912 & 0.88200962 & -5.07316585 \\ \mathrm{H} & 3.75774964 & -2.71422517 & -0.23156619 \\ \mathrm{~N} & 3.51662248 & -0.71168586 & -1.00132427 \\ \mathrm{C} & 4.14550646 & -1.70696845 & -0.27492517 \\ \mathrm{C} & 4.25693323 & 0.37398763 & -0.83876948 \\ \mathrm{H} & 4.06458389 & 1.34613957 & -1.26923017 \\ \mathrm{~N} & 5.32625491 & 0.11791260 & -0.04016142 \\ \mathrm{H} & 6.03812525 & 0.78212598 & 0.23050613 \\ \mathrm{C} & 5.27129093 & -1.20936124 & 0.32988944 \\ \mathrm{H} & 6.01378281 & -1.66394697 & 0.96670721 \\ & & & \\ & & & \\ & & & \end{array}$




$\begin{array}{crrc}\text { ModelA_PC } & & & \\ \text { C } & 0.00000000 & 0.00000000 & 0.00000000 \\ \text { H } & 1.09397763 & 0.00000000 & 0.00000000 \\ \text { H } & -0.36613380 & 1.02919684 & 0.00000000 \\ \text { N } & -0.53360040 & -0.73505995 & -1.15339335 \\ \text { H } & -0.94807981 & -1.63927946 & -0.96562930 \\ \text { C } & -0.46803920 & -0.35212245 & -2.43563341 \\ \text { N } & -0.70973745 & -1.36821490 & -6.10451169 \\ \text { H } & -0.65483273 & -2.37107639 & -6.27746374 \\ \text { H } & 0.15347168 & -0.95366689 & -6.48785875 \\ \text { N } & -1.11070095 & -1.02940605 & -3.37866497 \\ \text { H } & -0.90015249 & -1.02462692 & -4.42807276 \\ \text { H } & -1.72194293 & -1.78324099 & -3.08342334 \\ \text { H } & -0.35095768 & -0.49905497 & 0.90304226 \\ \text { C } & -0.03070639 & 1.58012548 & -4.44619085 \\ \text { H } & -1.11561167 & 1.51971539 & -4.54229931 \\ \text { H } & 0.27791716 & 2.62503277 & -4.51991351 \\ \text { S } & 0.48579194 & 1.08159956 & -2.76302455 \\ \text { H } & -1.48459055 & -1.02575224 & -6.67084504 \\ \text { H } & 0.48099615 & 0.99548063 & -5.21098571 \\ \text { H } & 3.29338606 & -0.92709965 & -5.68223756 \\ \text { N } & 1.89680892 & -0.05980866 & -7.07929027 \\ \text { C } & 3.15336664 & -0.41254540 & -6.62169996 \\ \text { C } & 2.09705340 & 0.53605394 & -8.24349479 \\ \text { H } & 1.33285846 & 0.94002014 & -8.89203680 \\ \text { N } & 3.42188061 & 0.58295484 & -8.54917838 \\ \text { H } & 3.82591203 & 0.98309849 & -9.38429696 \\ \text { C } & 4.11347024 & -0.02139860 & -7.52045061 \\ \text { H } & 5.18798292 & -0.11632873 & -7.52325783\end{array}$




\begin{tabular}{|c|c|c|c|}
\hline \multicolumn{4}{|c|}{ ModelB_RC } \\
\hline $\mathrm{C}$ & 0.00000000 & 0.00000000 & 0.00000000 \\
\hline $\mathrm{H}$ & 1.09643140 & 0.00000000 & 0.00000000 \\
\hline $\mathrm{H}$ & -0.35098293 & 1.03352873 & 0.00000000 \\
\hline $\mathrm{N}$ & -0.55781464 & -0.70997530 & -1.14343164 \\
\hline $\mathrm{H}$ & -0.69829127 & -1.70400083 & -1.03659547 \\
\hline $\mathrm{C}$ & -0.38036978 & -0.26997767 & -2.42659389 \\
\hline $\mathrm{N}$ & 0.26524717 & 0.82742051 & -2.68274289 \\
\hline $\mathrm{H}$ & 0.20723897 & 1.06479080 & -3.67138742 \\
\hline $\mathrm{H}$ & 1.46903449 & 1.96423415 & -1.83004968 \\
\hline $\mathrm{N}$ & -0.93092708 & -1.12090342 & -3.38361276 \\
\hline $\mathrm{H}$ & -0.93186942 & -0.76438120 & -4.32884730 \\
\hline $\mathrm{H}$ & -1.80654546 & -1.56279148 & -3.13292169 \\
\hline $\mathrm{H}$ & -0.35886157 & -0.48386372 & 0.91049062 \\
\hline $\mathrm{C}$ & -2.51957748 & 4.85740173 & -1.35496921 \\
\hline $\mathrm{H}$ & -2.36364503 & 5.27799973 & -2.35028662 \\
\hline $\mathrm{H}$ & -3.46516917 & 5.23461376 & -0.95988994 \\
\hline$S$ & -2.70201041 & 3.02793722 & -1.43265985 \\
\hline $\mathrm{H}$ & -1.47120675 & 2.73292152 & -1.90252693 \\
\hline $\mathrm{H}$ & -1.71280524 & 5.15780862 & -0.68475163 \\
\hline $\mathrm{H}$ & 4.02899166 & 1.61553373 & -1.69673013 \\
\hline $\mathrm{N}$ & 2.13853137 & 2.59646956 & -1.35584034 \\
\hline C & 3.50689741 & 2.45459540 & -1.26341660 \\
\hline $\mathrm{C}$ & 1.80854046 & 3.74558044 & -0.72024756 \\
\hline $\mathrm{H}$ & 0.80275953 & 4.12766042 & -0.62564641 \\
\hline$N$ & 2.87949924 & 4.34744191 & -0.22255575 \\
\hline $\mathrm{H}$ & 2.60596650 & 5.76239425 & 0.57662235 \\
\hline C & 3.95130291 & 3.54752832 & -0.55648194 \\
\hline $\mathrm{H}$ & 4.96149329 & 3.80406606 & -0.27261558 \\
\hline C & 1.09926871 & 6.91727032 & 1.03496235 \\
\hline 0 & 2.39689276 & 6.63661801 & 1.06976695 \\
\hline $\mathrm{O}$ & 0.25702840 & 6.21824881 & 0.48086106 \\
\hline C & 0.76483071 & 8.20293428 & 1.75923907 \\
\hline $\mathrm{H}$ & 1.06887228 & 8.12618539 & 2.80792606 \\
\hline $\mathrm{H}$ & 1.32540391 & 9.03382611 & 1.31973343 \\
\hline $\mathrm{H}$ & -0.30543526 & 8.39965606 & 1.69596669 \\
\hline
\end{tabular}




\begin{tabular}{|c|c|c|c|}
\hline \multicolumn{4}{|c|}{ ModelB_TS1 } \\
\hline $\mathrm{C}$ & 0.00000000 & 0.00000000 & 0.00000000 \\
\hline $\mathrm{H}$ & 1.09009347 & 0.00000000 & 0.00000000 \\
\hline $\mathrm{H}$ & -0.36856303 & 1.03141231 & 0.00000000 \\
\hline $\mathrm{N}$ & -0.49696385 & -0.71477595 & -1.17770152 \\
\hline $\mathrm{H}$ & -1.46842527 & -0.99083486 & -1.11468569 \\
\hline $\mathrm{C}$ & -0.07791852 & -0.44262303 & -2.42148520 \\
\hline $\mathrm{N}$ & 1.09369820 & 0.25882045 & -2.60611887 \\
\hline $\mathrm{H}$ & 1.39450576 & 0.22592361 & -3.57651662 \\
\hline $\mathrm{H}$ & 2.87748182 & -0.46183506 & -1.70682138 \\
\hline $\mathrm{N}$ & -0.61505714 & -1.12539066 & -3.46548391 \\
\hline $\mathrm{H}$ & -0.60673875 & -0.64657576 & -4.35734886 \\
\hline $\mathrm{H}$ & -1.45636199 & -1.66152269 & -3.29957106 \\
\hline $\mathrm{H}$ & -0.35218814 & -0.52740148 & 0.88742204 \\
\hline $\mathrm{C}$ & -1.18278491 & 2.36672788 & -4.29723111 \\
\hline $\mathrm{H}$ & -0.48794009 & 1.65901705 & -4.78243649 \\
\hline $\mathrm{H}$ & -2.19737124 & 2.12008166 & -4.62931554 \\
\hline$S$ & -1.06655216 & 2.34899219 & -2.45986629 \\
\hline $\mathrm{H}$ & 0.84067251 & 1.26292990 & -2.37006855 \\
\hline $\mathrm{H}$ & -0.93616049 & 3.35875884 & -4.68797511 \\
\hline $\mathrm{H}$ & 3.22512472 & -2.93970760 & -1.15134133 \\
\hline $\mathrm{N}$ & 3.73493767 & -0.84580104 & -1.31195699 \\
\hline C & 3.96799970 & -2.17192807 & -1.00042845 \\
\hline $\mathrm{C}$ & 4.86180130 & -0.14793683 & -1.00934594 \\
\hline $\mathrm{H}$ & 4.98434361 & 0.91749400 & -1.14675991 \\
\hline$N$ & 5.79529781 & -0.94879498 & -0.52272178 \\
\hline $\mathrm{H}$ & 7.27230122 & -0.21819952 & -0.10307101 \\
\hline C & 5.25021658 & -2.21492150 & -0.51154780 \\
\hline $\mathrm{H}$ & 5.80799590 & -3.06921350 & -0.15695057 \\
\hline C & 8.03907939 & 1.56061137 & -0.14543616 \\
\hline 0 & 8.13436273 & 0.26000846 & 0.13406800 \\
\hline $\mathrm{O}$ & 7.04101858 & 2.08467717 & -0.62275996 \\
\hline C & 9.30223638 & 2.31828941 & 0.19497287 \\
\hline $\mathrm{H}$ & 9.51553504 & 2.22074329 & 1.26422696 \\
\hline $\mathrm{H}$ & 10.15143498 & 1.88866827 & -0.34552531 \\
\hline $\mathrm{H}$ & 9.18583410 & 3.36999822 & -0.06635356 \\
\hline
\end{tabular}




\begin{tabular}{|c|c|c|c|}
\hline \multicolumn{4}{|c|}{ ModelB_INT } \\
\hline $\mathrm{C}$ & 0.00000000 & 0.00000000 & 0.00000000 \\
\hline $\mathrm{H}$ & 1.09382490 & 0.00000000 & 0.00000000 \\
\hline $\mathrm{H}$ & -0.35607754 & 1.03999221 & 0.00000000 \\
\hline $\mathrm{N}$ & -0.50012026 & -0.80423519 & -1.12323581 \\
\hline $\mathrm{H}$ & -1.48037407 & -1.03530919 & -0.98764207 \\
\hline $\mathrm{C}$ & -0.32005115 & -0.29901179 & -2.47448741 \\
\hline $\mathrm{N}$ & 1.09574463 & -0.03805833 & -2.69130264 \\
\hline $\mathrm{H}$ & 1.24619593 & 0.13474507 & -3.68181696 \\
\hline $\mathrm{H}$ & 2.11771111 & -1.63831297 & -2.07441308 \\
\hline $\mathrm{N}$ & -0.72680338 & -1.25195765 & -3.48277516 \\
\hline $\mathrm{H}$ & -1.73425242 & -1.37438266 & -3.48800576 \\
\hline $\mathrm{H}$ & -0.27844755 & -2.15076256 & -3.31158907 \\
\hline $\mathrm{H}$ & -0.32076139 & -0.47721374 & 0.92927646 \\
\hline $\mathrm{C}$ & -1.00570950 & 1.79281455 & -4.38257878 \\
\hline $\mathrm{H}$ & 0.00215908 & 2.21408877 & -4.42932652 \\
\hline $\mathrm{H}$ & -1.10450671 & 0.96683969 & -5.08909404 \\
\hline$S$ & -1.42618481 & 1.24374912 & -2.68856667 \\
\hline $\mathrm{H}$ & 1.40689145 & 0.78268827 & -2.17771319 \\
\hline $\mathrm{H}$ & -1.72088973 & 2.57743753 & -4.64090832 \\
\hline $\mathrm{H}$ & 1.23258214 & -3.35373327 & -0.39705021 \\
\hline $\mathrm{N}$ & 2.63614505 & -2.47170597 & -1.77060557 \\
\hline C & 2.20084428 & -3.42048932 & -0.86763693 \\
\hline $\mathrm{C}$ & 3.87800004 & -2.83542993 & -2.17925044 \\
\hline $\mathrm{H}$ & 4.48056336 & -2.28610828 & -2.88892099 \\
\hline$N$ & 4.26189887 & -3.95911688 & -1.59242100 \\
\hline $\mathrm{H}$ & 5.77358712 & -4.53676099 & -1.99503163 \\
\hline C & 3.22137180 & -4.33523529 & -0.76969714 \\
\hline $\mathrm{H}$ & 3.27119629 & -5.22959661 & -0.16591287 \\
\hline C & 7.25892642 & -4.03024077 & -3.14483165 \\
\hline 0 & 6.70285338 & -4.86062893 & -2.26546593 \\
\hline $\mathrm{O}$ & 6.71948367 & -3.01430292 & -3.56631361 \\
\hline C & 8.63870609 & -4.48069823 & -3.57188404 \\
\hline $\mathrm{H}$ & 9.28819319 & -4.57246557 & -2.69568658 \\
\hline $\mathrm{H}$ & 8.57822922 & -5.47064876 & -4.03530977 \\
\hline $\mathrm{H}$ & 9.06161954 & -3.76529279 & -4.27725631 \\
\hline
\end{tabular}




$\begin{array}{lrrc}\text { ModelB_TS2 } & & & \\ \text { C } & 0.00000000 & 0.00000000 & 0.00000000 \\ \text { H } & 1.09654853 & 0.00000000 & 0.00000000 \\ \text { H } & -0.36058792 & 1.03287836 & 0.00000000 \\ \text { N } & -0.53341799 & -0.72968573 & -1.15362472 \\ \text { H } & -0.83181445 & -1.69221419 & -0.97614960 \\ \text { C } & -0.21210685 & -0.48131019 & -2.43308751 \\ \text { N } & 1.46745860 & -1.48075912 & -2.81562871 \\ \text { H } & 1.63432587 & -1.71841476 & -3.79164229 \\ \text { H } & 1.33601296 & -2.38233180 & -2.24887505 \\ \text { N } & -1.02061245 & -1.15680813 & -3.35782319 \\ \text { H } & -0.81659025 & -0.98374013 & -4.33212048 \\ \text { H } & -2.00838185 & -1.06103820 & -3.15082673 \\ \text { H } & -0.34391064 & -0.49337132 & 0.91127692 \\ \text { C } & 0.50078377 & 1.28402844 & -4.52221373 \\ \text { H } & -0.44946909 & 1.12318505 & -5.03738359 \\ \text { H } & 0.84728452 & 2.29368509 & -4.75367279 \\ \text { S } & 0.31482220 & 1.24778022 & -2.71036321 \\ \text { H } & 2.27359767 & -0.96643367 & -2.46572083 \\ \text { H } & 1.25626277 & 0.57462189 & -4.86943122 \\ \text { H } & -0.96611223 & -4.24610748 & -2.10423854 \\ \text { N } & 0.86070081 & -3.60139972 & -1.15741366 \\ \text { C } & -0.45878866 & -4.00768611 & -1.17835465 \\ \text { C } & 1.12829159 & -3.44301964 & 0.15408843 \\ \text { H } & 2.09388849 & -3.12046551 & 0.52569333 \\ \text { N } & 0.09226664 & -3.70587902 & 0.96940976 \\ \text { H } & -0.03682666 & -3.37754533 & 2.44182669 \\ \text { C } & -0.92770256 & -4.06916598 & 0.12434618 \\ \text { H } & -1.89971699 & -4.36131618 & 0.49954313 \\ \text { C } & -0.47738140 & -1.97312082 & 3.80190242 \\ \text { O } & -0.13103025 & -3.20599475 & 3.49056169 \\ \text { O } & -0.66824775 & -1.06736618 & 2.99024513 \\ \text { C } & -0.62468666 & -1.76412389 & 5.29743406 \\ \text { H } & -1.38777130 & -2.44273606 & 5.69208601 \\ \text { H } & -2.00945139 & 5.80105410 \\ \text { H } & -0.73058747 & 5.50774926\end{array}$




\begin{tabular}{|c|c|c|c|}
\hline C & 0.00000000 & 0.00000000 & 0.00000000 \\
\hline $\mathrm{H}$ & 1.09598939 & 0.00000000 & 0.00000000 \\
\hline $\mathrm{H}$ & -0.35306593 & 1.03644638 & 0.00000000 \\
\hline $\mathrm{N}$ & -0.54100766 & -0.74901981 & -1.12524869 \\
\hline $\mathrm{H}$ & -1.08648926 & -1.64542014 & -0.95792180 \\
\hline $\mathrm{C}$ & -0.46755841 & -0.38917958 & -2.39894467 \\
\hline $\mathrm{N}$ & -0.58270168 & -1.44791145 & -6.24963563 \\
\hline $\mathrm{H}$ & -0.98862727 & -0.84229166 & -6.96096850 \\
\hline $\mathrm{H}$ & -1.11049784 & -2.33457292 & -6.29255584 \\
\hline$N$ & -1.10061347 & -1.09496026 & -3.33086051 \\
\hline $\mathrm{H}$ & -0.88058861 & -1.04706872 & -4.33990061 \\
\hline $\mathrm{H}$ & -1.62600672 & -1.91075821 & -2.98789403 \\
\hline $\mathrm{H}$ & -0.34157707 & -0.48609144 & 0.91492499 \\
\hline C & -0.09539978 & 1.49360780 & -4.45919393 \\
\hline $\mathrm{H}$ & -1.17147141 & 1.67445640 & -4.45407864 \\
\hline $\mathrm{H}$ & 0.42934655 & 2.42068009 & -4.69950936 \\
\hline $\mathrm{S}$ & 0.48686321 & 1.05286297 & -2.78379931 \\
\hline $\mathrm{H}$ & 0.36351000 & -1.66116766 & -6.56095955 \\
\hline $\mathrm{H}$ & 0.15852998 & 0.72444891 & -5.18775904 \\
\hline $\mathrm{H}$ & -4.06574648 & -3.83919413 & -7.14428590 \\
\hline $\mathrm{N}$ & -2.28531269 & -3.95333362 & -5.93912900 \\
\hline C & -3.64499645 & -4.01948807 & -6.16479484 \\
\hline C & -2.14190633 & -4.23783369 & -4.64900453 \\
\hline $\mathrm{H}$ & -1.20349269 & -4.27858328 & -4.11406230 \\
\hline $\mathrm{N}$ & -3.32651737 & -4.47669062 & -4.03918099 \\
\hline $\mathrm{H}$ & -3.43629809 & -4.59768025 & -3.00366311 \\
\hline C & -4.30266716 & -4.33885054 & -4.99619300 \\
\hline $\mathrm{H}$ & -5.34934969 & -4.47608236 & -4.77120993 \\
\hline C & -2.71552288 & -3.76891383 & -0.74505863 \\
\hline $\mathrm{O}$ & -3.42985563 & -4.62143084 & -1.31912262 \\
\hline $\mathrm{O}$ & -1.97910984 & -2.90599679 & -1.34839854 \\
\hline C & -2.70368310 & -3.73417594 & 0.78226091 \\
\hline $\mathrm{H}$ & -3.08744628 & -2.76866213 & 1.13160561 \\
\hline $\mathrm{H}$ & -3.31903273 & -4.53555062 & 1.19384143 \\
\hline $\mathrm{H}$ & -1.67682421 & -3.83429418 & 1.15061802 \\
\hline
\end{tabular}




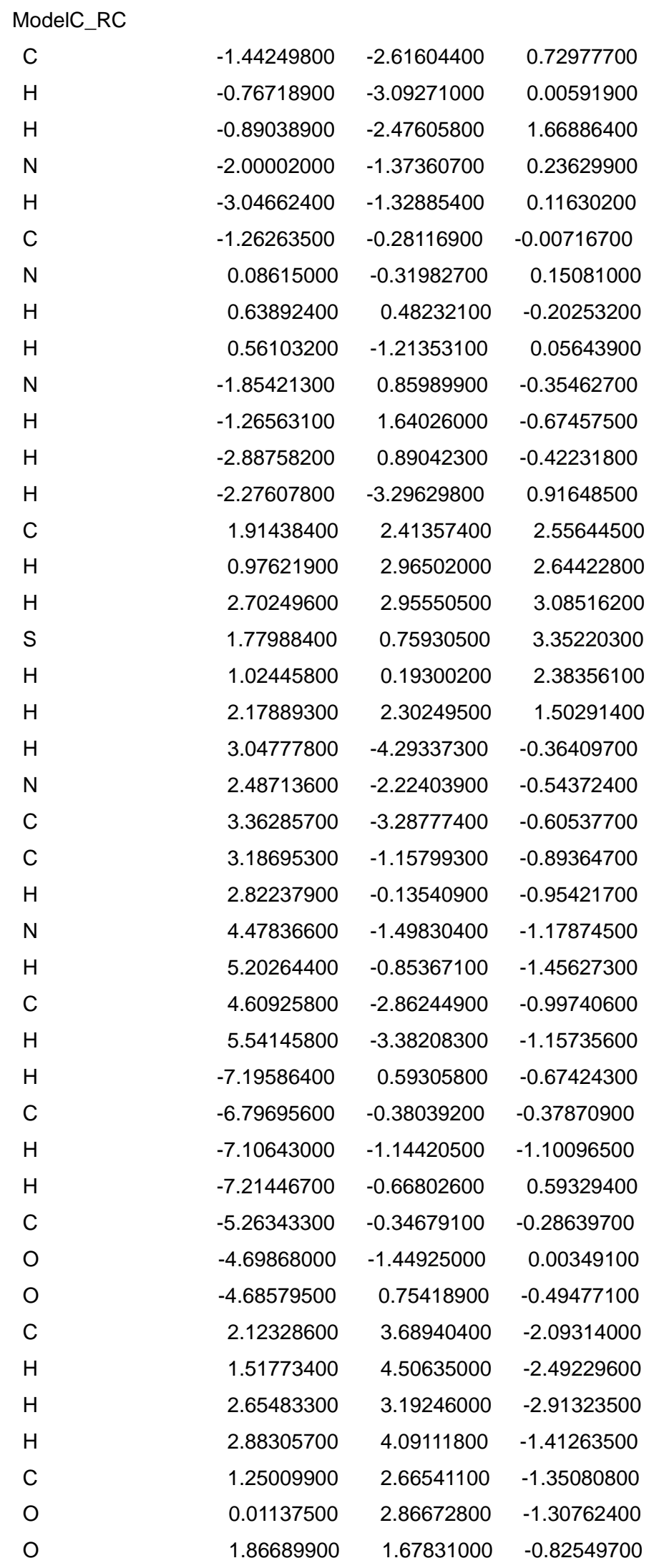




\begin{tabular}{|c|c|c|c|}
\hline Mod & & & \\
\hline C & 1.45155700 & 0.21618300 & 2.59901200 \\
\hline $\mathrm{H}$ & 0.60136600 & 0.69878400 & 3.09739500 \\
\hline $\mathrm{H}$ & 1.62064200 & -0.76571600 & 3.06215600 \\
\hline $\mathrm{N}$ & 1.24224900 & 0.10804600 & 1.16703200 \\
\hline $\mathrm{H}$ & 2.12990200 & 0.04159100 & 0.61943600 \\
\hline C & 0.19000300 & -0.60882200 & 0.64732100 \\
\hline $\mathrm{N}$ & -1.14898500 & -0.14541000 & 1.08858300 \\
\hline $\mathrm{H}$ & -2.05769700 & -0.49216300 & 0.43973300 \\
\hline $\mathrm{H}$ & -1.15946600 & 0.89240200 & 1.09079300 \\
\hline$N$ & 0.24909000 & -0.95516100 & -0.64155700 \\
\hline $\mathrm{H}$ & -0.60689000 & -1.18675000 & -1.15917100 \\
\hline $\mathrm{H}$ & 1.18227700 & -1.06235600 & -1.09297000 \\
\hline $\mathrm{H}$ & 2.33230800 & 0.84395000 & 2.75789800 \\
\hline$C$ & -0.43143000 & -3.76834800 & 0.71011200 \\
\hline $\mathrm{H}$ & -0.03222900 & -3.39722000 & -0.24161400 \\
\hline $\mathrm{H}$ & -0.00468000 & -4.75885400 & 0.89794600 \\
\hline$S$ & 0.04538700 & -2.63064100 & 2.06163900 \\
\hline $\mathrm{H}$ & -1.31718000 & -0.52883700 & 2.02070400 \\
\hline $\mathrm{H}$ & -1.51803700 & -3.86257000 & 0.61618000 \\
\hline $\mathrm{H}$ & 1.08515300 & 2.52647800 & 0.26877500 \\
\hline$N$ & -0.97894400 & 2.86394700 & 0.74552300 \\
\hline $\mathrm{C}$ & 0.24756600 & 3.20831000 & 0.20952500 \\
\hline$C$ & -1.78745300 & 3.87080600 & 0.48445500 \\
\hline $\mathrm{H}$ & -2.83353100 & 3.93090400 & 0.74966100 \\
\hline$N$ & -1.13647300 & 4.86204500 & -0.19250900 \\
\hline $\mathrm{H}$ & -1.54191200 & 5.72450100 & -0.52195700 \\
\hline $\mathrm{C}$ & 0.17108100 & 4.44635900 & -0.37701600 \\
\hline $\mathrm{H}$ & 0.89819600 & 5.04542600 & -0.90249900 \\
\hline $\mathrm{H}$ & 5.71236500 & 0.37767600 & -1.69371900 \\
\hline $\mathrm{C}$ & 5.08748100 & -0.46483600 & -2.00315000 \\
\hline $\mathrm{H}$ & 5.60399800 & -1.39424900 & -1.73140000 \\
\hline $\mathrm{H}$ & 4.95316400 & -0.46773800 & -3.08919700 \\
\hline$C$ & 3.72543200 & -0.43694000 & -1.28816100 \\
\hline $\mathrm{O}$ & 3.66085300 & 0.21466900 & -0.20187100 \\
\hline $\mathrm{O}$ & 2.78810400 & -1.09066200 & -1.82685300 \\
\hline$C$ & -4.65719000 & -1.52019500 & -1.92221600 \\
\hline $\mathrm{H}$ & -4.58941400 & -1.71162700 & -2.99531400 \\
\hline $\mathrm{H}$ & -5.34151500 & -0.68917000 & -1.72422200 \\
\hline $\mathrm{H}$ & -5.06854200 & -2.40741900 & -1.42653100 \\
\hline C & -3.27501900 & -1.22380300 & -1.33917200 \\
\hline $\mathrm{O}$ & -2.25808400 & -1.47084200 & -2.01791700 \\
\hline O & -3.27777300 & -0.74233200 & -0.14361700 \\
\hline
\end{tabular}




\begin{tabular}{|c|c|c|c|}
\hline \multicolumn{4}{|c|}{ ModelC_INT } \\
\hline C & -1.26129000 & -1.73795500 & -2.58577800 \\
\hline $\mathrm{H}$ & -0.55992200 & -1.46387300 & -3.38675600 \\
\hline $\mathrm{H}$ & -1.20657800 & -2.82930600 & -2.44928400 \\
\hline $\mathrm{N}$ & -1.02158100 & -0.97987400 & -1.37361400 \\
\hline $\mathrm{H}$ & -1.84519000 & -0.92992700 & -0.74708100 \\
\hline C & 0.20866900 & -1.03871700 & -0.67649500 \\
\hline $\mathrm{N}$ & 1.18660000 & -0.03062700 & -1.28668900 \\
\hline $\mathrm{H}$ & 2.14529200 & 0.09324900 & -0.75010500 \\
\hline $\mathrm{H}$ & 0.69528200 & 0.88624600 & -1.30620600 \\
\hline $\mathrm{N}$ & 0.06515600 & -0.71026600 & 0.65591500 \\
\hline $\mathrm{H}$ & 0.89882500 & -0.45045000 & 1.18504800 \\
\hline $\mathrm{H}$ & -0.74212300 & -1.11126700 & 1.15578600 \\
\hline $\mathrm{H}$ & -2.26779400 & -1.48767400 & -2.93366700 \\
\hline C & 2.25865700 & -2.88547500 & 0.35329700 \\
\hline $\mathrm{H}$ & 1.71064700 & -2.72239000 & 1.28439500 \\
\hline $\mathrm{H}$ & 2.67827400 & -3.89567900 & 0.35835200 \\
\hline $\mathrm{S}$ & 1.11362500 & -2.80455100 & -1.06641400 \\
\hline $\mathrm{H}$ & 1.40763900 & -0.31972300 & -2.23822900 \\
\hline $\mathrm{H}$ & 3.06441600 & -2.15376700 & 0.27192500 \\
\hline $\mathrm{H}$ & -2.11339000 & 1.40445200 & -0.51761500 \\
\hline $\mathrm{N}$ & -0.36704100 & 2.54269900 & -1.09017300 \\
\hline C & -1.62048100 & 2.36834000 & -0.53067100 \\
\hline C & -0.04770400 & 3.80553900 & -0.89257200 \\
\hline $\mathrm{H}$ & 0.87684500 & 4.28142700 & -1.18812200 \\
\hline $\mathrm{N}$ & -1.04461800 & 4.46888400 & -0.23656900 \\
\hline $\mathrm{H}$ & -1.02690200 & 5.43350500 & 0.05578000 \\
\hline C & -2.05740600 & 3.55429600 & 0.00315400 \\
\hline $\mathrm{H}$ & -2.96094400 & 3.81860300 & 0.52984200 \\
\hline $\mathrm{H}$ & -5.44404800 & -0.40244400 & 1.64305900 \\
\hline C & -4.76653300 & -1.18315400 & 2.00231700 \\
\hline $\mathrm{H}$ & -5.22731700 & -2.15627800 & 1.78891600 \\
\hline $\mathrm{H}$ & -4.63246000 & -1.10667100 & 3.08562200 \\
\hline C & -3.40743700 & -1.11269800 & 1.27791700 \\
\hline $\mathrm{O}$ & -3.41312500 & -0.61595400 & 0.10807600 \\
\hline $\mathrm{O}$ & -2.40863000 & -1.57855900 & 1.89027000 \\
\hline C & 4.89326100 & 1.12335500 & 1.62070500 \\
\hline $\mathrm{H}$ & 4.84495300 & 1.25620100 & 2.70399400 \\
\hline $\mathrm{H}$ & 5.12948400 & 2.07808300 & 1.13751800 \\
\hline $\mathrm{H}$ & 5.70326700 & 0.42788100 & 1.37201200 \\
\hline C & 3.56628800 & 0.57794800 & 1.07641100 \\
\hline $\mathrm{O}$ & 2.65183000 & 0.29528600 & 1.88083800 \\
\hline $\mathrm{O}$ & 3.51092400 & 0.44398000 & -0.19973200 \\
\hline
\end{tabular}




\begin{tabular}{|c|c|c|c|}
\hline \multicolumn{4}{|c|}{ Model_TS2 } \\
\hline C & -0.31988300 & -2.53104600 & -2.22380100 \\
\hline $\mathrm{H}$ & -0.24847300 & -1.69542800 & -2.94094900 \\
\hline $\mathrm{H}$ & 0.41627200 & -3.29008500 & -2.51451500 \\
\hline $\mathrm{N}$ & -0.16002400 & -2.12663600 & -0.84301800 \\
\hline $\mathrm{H}$ & -1.05654200 & -1.89778600 & -0.36840100 \\
\hline $\mathrm{C}$ & 0.94608000 & -1.44874500 & -0.39043800 \\
\hline $\mathrm{N}$ & 0.93600700 & 0.24441200 & -1.17927800 \\
\hline $\mathrm{H}$ & 1.68237700 & 0.86950900 & -0.77709600 \\
\hline $\mathrm{H}$ & 0.01591200 & 0.68322200 & -1.03760700 \\
\hline $\mathrm{N}$ & 0.97271800 & -1.21531200 & 0.95954800 \\
\hline $\mathrm{H}$ & 1.54685600 & -0.42959300 & 1.28851300 \\
\hline $\mathrm{H}$ & 0.06151600 & -1.31762900 & 1.43189600 \\
\hline $\mathrm{H}$ & -1.31640800 & -2.96868100 & -2.32178200 \\
\hline $\mathrm{C}$ & 3.78555700 & -1.36709600 & -0.10145900 \\
\hline $\mathrm{H}$ & 3.61281700 & -1.62780000 & 0.94382500 \\
\hline $\mathrm{H}$ & 4.73404000 & -1.79475700 & -0.43879400 \\
\hline $\mathrm{S}$ & 2.50777900 & -2.13092400 & -1.15700000 \\
\hline $\mathrm{H}$ & 1.10097300 & 0.16096400 & -2.18005700 \\
\hline $\mathrm{H}$ & 3.80300300 & -0.28200000 & -0.21660300 \\
\hline $\mathrm{H}$ & -3.09847100 & 0.28032300 & -0.50487800 \\
\hline $\mathrm{N}$ & -1.74225500 & 1.82406600 & -1.14396400 \\
\hline $\mathrm{C}$ & -2.96086600 & 1.33866800 & -0.70569900 \\
\hline C & -1.87914200 & 3.13012400 & -1.24017500 \\
\hline $\mathrm{H}$ & -1.11051900 & 3.82849100 & -1.54105100 \\
\hline$N$ & -3.14087000 & 3.52177000 & -0.89083200 \\
\hline $\mathrm{H}$ & -3.48426700 & 4.46917700 & -0.85964200 \\
\hline C & -3.84307100 & 2.37880500 & -0.54353500 \\
\hline $\mathrm{H}$ & -4.86908700 & 2.41444500 & -0.21115000 \\
\hline $\mathrm{H}$ & -4.64829100 & -1.10396800 & 2.12407500 \\
\hline $\mathrm{C}$ & -4.03183100 & -2.00698600 & 2.21818300 \\
\hline $\mathrm{H}$ & -4.57971500 & -2.82593000 & 1.73954900 \\
\hline $\mathrm{H}$ & -3.88019900 & -2.22323700 & 3.27888900 \\
\hline C & -2.68803000 & -1.77651900 & 1.50217000 \\
\hline $\mathrm{O}$ & -2.73755500 & -1.71206300 & 0.22872900 \\
\hline $\mathrm{O}$ & -1.65901300 & -1.65243000 & 2.21149300 \\
\hline C & 3.61688800 & 3.27942400 & 1.66347700 \\
\hline $\mathrm{H}$ & 3.85292200 & 3.12571100 & 2.71963600 \\
\hline $\mathrm{H}$ & 2.92164200 & 4.12342300 & 1.57419900 \\
\hline $\mathrm{H}$ & 4.52196200 & 3.54449000 & 1.10632600 \\
\hline $\mathrm{C}$ & 2.96353500 & 2.02647100 & 1.05394400 \\
\hline $\mathrm{O}$ & 2.57456400 & 1.12432800 & 1.83942700 \\
\hline $\mathrm{O}$ & 2.85985000 & 2.01206700 & -0.21659600 \\
\hline
\end{tabular}




\begin{tabular}{|c|c|c|c|}
\hline $\mathrm{C}$ & -0.76074400 & -3.17716700 & -1.93447000 \\
\hline $\mathrm{H}$ & -0.42521700 & -2.66455800 & -2.84502400 \\
\hline $\mathrm{H}$ & -0.22594000 & -4.13251300 & -1.85705300 \\
\hline $\mathrm{N}$ & -0.58562300 & -2.35814700 & -0.74947000 \\
\hline $\mathrm{H}$ & -1.47778300 & -2.04155800 & -0.27283700 \\
\hline C & 0.58232500 & -1.86826400 & -0.32196000 \\
\hline $\mathrm{N}$ & 1.78491500 & 1.71506700 & -2.47716500 \\
\hline $\mathrm{H}$ & 2.19310500 & 1.63739700 & -1.54133100 \\
\hline $\mathrm{H}$ & 0.77437800 & 1.61949000 & -2.37894700 \\
\hline $\mathrm{N}$ & 0.65090300 & -1.14528300 & 0.77615800 \\
\hline $\mathrm{H}$ & 1.48846200 & -0.60061200 & 1.11517100 \\
\hline $\mathrm{H}$ & -0.23261900 & -1.03386200 & 1.31125100 \\
\hline $\mathrm{H}$ & -1.82909200 & -3.38342600 & -2.02645100 \\
\hline $\mathrm{C}$ & 3.40249400 & -1.51453100 & -0.45151000 \\
\hline $\mathrm{H}$ & 3.49717500 & -1.88691900 & 0.56910200 \\
\hline $\mathrm{H}$ & 4.27145800 & -1.81713200 & -1.04217300 \\
\hline $\mathrm{S}$ & 1.99563300 & -2.30088900 & -1.30607100 \\
\hline $\mathrm{H}$ & 1.96706900 & 2.66369200 & -2.79472400 \\
\hline $\mathrm{H}$ & 3.31335900 & -0.42506100 & -0.44891400 \\
\hline $\mathrm{H}$ & -2.73976300 & 0.48764400 & -0.81142600 \\
\hline $\mathrm{N}$ & -1.61196900 & 2.07998900 & -1.75042100 \\
\hline C & -2.55735100 & 1.55179200 & -0.89133300 \\
\hline $\mathrm{C}$ & -1.61948200 & 3.37823900 & -1.53432400 \\
\hline $\mathrm{H}$ & -0.98723300 & 4.10670700 & -2.02256200 \\
\hline $\mathrm{N}$ & -2.53655900 & 3.72347900 & -0.57954100 \\
\hline $\mathrm{H}$ & -2.69764100 & 4.64824300 & -0.21182100 \\
\hline C & -3.14051400 & 2.55363200 & -0.15424700 \\
\hline $\mathrm{H}$ & -3.88469400 & 2.53927100 & 0.62683500 \\
\hline $\mathrm{H}$ & -4.93754100 & -0.63728800 & 1.71457500 \\
\hline $\mathrm{C}$ & -4.24069100 & -1.27045400 & 2.27469100 \\
\hline $\mathrm{H}$ & -4.68784900 & -2.27021700 & 2.32818300 \\
\hline $\mathrm{H}$ & -4.10758300 & -0.88003200 & 3.28647300 \\
\hline $\mathrm{C}$ & -2.89820600 & -1.35584900 & 1.53460700 \\
\hline $\mathrm{O}$ & -2.96141100 & -1.66325100 & 0.29712900 \\
\hline $\mathrm{O}$ & -1.84617000 & -1.13035700 & 2.18443500 \\
\hline C & 3.83703700 & 2.27808600 & 2.53642000 \\
\hline $\mathrm{H}$ & 4.39100200 & 1.68591700 & 3.27277700 \\
\hline $\mathrm{H}$ & 3.05507100 & 2.82121700 & 3.08138900 \\
\hline $\mathrm{H}$ & 4.50135300 & 3.00018100 & 2.05386500 \\
\hline $\mathrm{C}$ & 3.17305700 & 1.35329800 & 1.49916300 \\
\hline $\mathrm{O}$ & 2.57136400 & 0.33799900 & 1.97383000 \\
\hline $\mathrm{O}$ & 3.27011500 & 1.66801200 & 0.28356000 \\
\hline
\end{tabular}

